\title{
Introduction: Flavoprotein structure and mechanism
}

\author{
VINCENT MASSEY
}

Department of Biological Chemistry, University of Michigan, Ann Arbor, Michigan 48109, USA

UNDERSTANDING OF THE VERSATILE chemistry of flavins and the mechanisms of action of flavoprotein enzymes has progressed enormously in the last 10 years, especially since the X-ray crystal structures of many flavoenzymes have become available and meaningful site-directed mutagenesis of putative active site residues has been possible. This issue of The FASEB Journal contains the first in a series of articles that will attempt to review and summarize the present state of knowledge of representatives of the various classes of flavoenzymes. In this series, wherever possible, information regarding the catalytic mechanism obtained from solution studies will be correlated with structural information obtained by $\mathrm{X}$ ray crystallographic techniques.

It has been apparent for many years that flavoenzymes can be grouped into a relatively small number of classes, where members within the same class share many common properties differing from those of other classes, including the types of reactions catalyzed, the ability to use molecular oxygen as acceptor, and the nature of auxiliary redox centers. In this series, examples of most of these classes will be considered. The basis for classification and details of common properties have been reviewed previously (1) but for convenience will be reiterated here.

\section{SIMPLE FLAVOPROTEINS}

\section{Oxidases}

Simple flavoproteins are conveniently classified on the basis of the reactivity of the reduced enzyme with molecular oxygen. The oxidases, such as D-amino acid oxidase, glucose oxidase, glycolate oxidase, react very rapidly with $\mathrm{O}_{2}$ to yield $\mathrm{H}_{2} \mathrm{O}_{2}$ and oxidized flavoprotein in what appears to be a simple second-order process without observable intermediates (2). Enzymes of this class have also been found to share several common properties: they all stabilize the red anionic flavin radical on one-electron reduction, and they all stabilize a flavin N(5)-sulfite adduct and the benzoquinoid anion form of 6- and 8-substituted hydroxy- and mercaptoflavins, where the negative charge of the anionic flavin is localized in the $\mathrm{N}(1)-\mathrm{C}(2) \mathrm{O}$ region (3). These properties have been interpreted to indicate the presence of a positively charged locus of the protein interacting with the pyrimidine ring of the flavin. The X-ray crystal structures of several flavoprotein oxidases have been solved in recent years, e.g., glycolate oxidase (4), glucose oxidase (5), and cholesterol oxidase (6). Glycolate oxidase shows the expected positively charged residue Lys230, and both glucose oxidase and cholesterol oxidase have histidine residues in the vicinity of the flavin pyrimidine ring. Solution studies of oxidases, where the native flavin has been replaced by flavins with chemically reactive substituents, in most cases showed that the flavin was well buried in the intact protein, with only the region around the N(5)-position accessible to solvent (3). These findings are confirmed by the crystal structures so far available.
An unusual example of a flavoprotein oxidase, involving covalent linkage of the flavin to the protein via the flavin 8-methyl substituent, is monoamine oxidase. This enzyme has been studied extensively from a mechanistic viewpoint, and although so far little is known about its structure, because of its medical importance an article by T. P. Singer and R. Ramsay reviewing its properties will be included in this series.

\section{Electron transferases}

The enzymes of this class are all involved physiologically in. single-electron transfers, e.g., flavodoxin, ferredoxin-NADP reductase, and NADPH-cytochrome $\mathrm{P}-450$ reductase. These enzymes all react sluggishly with $\mathrm{O}_{2}$, and in the process produce $\mathrm{O}_{2}{ }^{-}$and the flavin semiquinone. The semiquinone is also significantly stabilized thermodynamically, generally as the blue neutral radical species. In contrast to the oxidases, this group shows no tendency to form flavin $\mathrm{N}(5)$ sulfite adducts or to stabilize the benzoquinoid forms of 6 and 8-substituted flavins. Solution studies with chemically reactive flavins such as 8-Cl-, 8-mercapto-, or 8-SCN-flavins revealed that the benzene ring of the flavin is the only part readily accessible to solvent (3). Again, these conclusions have received support from the crystal structures available, e.g., flavodoxin (7) and ferredoxin-NADP ${ }^{+}$reductase (8).

Acyl-CoA dehydrogenases are difficult to classify because the reaction of the enzyme with the acyl-CoA substrate involves the formation of an unsaturated $C=C$ structure apparently by concerted proton abstraction from the substrate $\alpha$-carbon by an enzyme base and by hydride transfer from the $\beta$-carbon to the flavin $N(5)$ (9). However, the catalytic reaction cycle is completed physiologically by sequential single-electron transfers to another flavoprotein, the electron transfer flavoprotein. The latter is unusual among electron transferases in stabilizing thermodynamically the flavin anionic semiquinone; the intermediate radical form of the dehydrogenase is the blue neutral semiquinone in the absence of enoyl-CoA product, and the red anionic semiquinone in the presence of product (10). The crystal structure of the medium chain acyl-CoA dehydrogenase from pig kidney has now been solved and the active site base identified. This enzyme will be discussed by C. Thorpe and J.-J. P. Kim in an early article of this series.

\section{Flavoprotein monooxygenases}

In this third main class of simple flavoproteins, the reduced enzyme reacts with $\mathrm{O}_{2}$ to form readily observable flavin $\mathrm{C}(4 \mathrm{a})$ hydroperoxide intermediates. With all enzymes of this class the physiological reductant is NADH or NADPH. In the absence of further substrate, the flavin hydroperoxide decays nonproductively to $\mathrm{H}_{2} \mathrm{O}_{2}$ and oxidized flavin. However, in the presence of the third substrate the flavin hydroperoxide reacts to transfer an oxygen atom to the substrate, resulting in a $\mathrm{C}(4 \mathrm{a})$-hydroxyflavin, which upon dehydration 
returns the flavin to its oxidized state for the next catalytic cycle. Two examples of such enzymes will be considered in this series. The first is $p$-hydroxybenzoate hydroxylase, where the aromatic substrate is hydroxylated at the position ortho to the activating para-substituent. This enzyme is the most thoroughly studied mechanistically, and recent work from two separate groups has presented evidence for substantial movement of the flavin within the protein during catalysis. This enzyme is reviewed jointly by members of both research groups in this issue (11). The second enzyme of this class is bacterial luciferase, where the third substrate is a long-chain aldehyde, and the products fatty acid and $\mathrm{H}_{2} \mathrm{O}$ are formed with accompanying emission of visible light. This enzyme will be discussed by T. O. Baldwin.

\section{FLAVOPROTEINS WITH AUXILIARY REDOX CENTERS}

\section{Flavoprotein-disulfide oxidoreductases}

Enzymes of this class contain a disulfide in close juxtaposition to the flavin (in all cases, FAD). Catalysis involves interaction of the flavin with a pyridine nucleotide, electron transfer through the flavin to the redox active disulfide/dithiol, and interaction of the latter with the second substrate (12). The second substrate is usually a disulfide/dithiol, e.g., a lipoic acid derivative in lipoamide dehydrogenase, glutathione in glutathione reductase, thioredoxin in thioredoxin reductase, and trypanothione in trypanothione reductase. An interesting variation of the family is found with mercuric reductase, which catalyzes the reduction of $\mathrm{Hg}^{2+}$ to $\mathrm{Hg}^{\mathrm{O}}$. In this enzyme the $\mathrm{Hg}^{2+}$ is coordinated with at least one protein cysteine residue in its interaction with the active-site disulfide-dithiol pair (12). A further variant of the family is found with streptococcal NADH oxidase and NADH peroxidase, which use a cysteine sulfenic acid in catalysis (13). Crystal structures of all the enzymes listed above have been determined and reviewed extensively (for an overview, see refs 12,14$)$. In the present series thioredoxin reductase and trypanothione reductase will be considered by $\mathrm{C}$. $\mathrm{H}$. Williams, Jr. and by R. L. Krauth-Siegel and R. Schöneck, respectively, and relationships between all enzymes of the class will be discussed later by $\mathrm{E}$. Pai.

\section{Heme-containing flavoproteins (flavocytochtomes)}

The best-known example of this class is yeast lactate dehydrogenase (flavocytochrome $b_{2}$ ), which, in addition to the FMN prosthetic group, also contains a b-type cytochrome domain. The crystal structure of this enzyme was solved some time ago (15) and correlation with the extensive mechanistic information available has been reviewed in detail (16).

Another interesting example of a heme-containing flavoenzyme is the fusion complex of cytochrome P-450 BM-3, where an FMN- and FAD-containing NADPHcytochrome $\mathrm{P}-450$ reductase forms one domain and a cytochrome P-450 another domain of a single catalytically self-sufficient protein (17).

Recent work with nitric oxide synthase has revealed that this enzyme, in addition to containing FAD and FMN, also contains a heme prosthetic group with cytochrome P-450-like characteristics. The enzyme also appears to involve a pterin cofactor in its function. This complex enzyme will also be reviewed in this series by $K$. A. White and $M$. A. Marletta.

\section{Metal-containing flavoproteins}

The longest-known example of a complex flavoprotein is xanthine oxidase, which in 1954 had already been shown to contain molybdenum and iron-sulfur centers in addition to the FAD prosthetic group. Extensive mechanistic work and flavin replacement studies have been carried out with both the oxidase and dehydrogenase forms of the enzyme from various sources. Although crystal structures are not yet available, considerable information concerning the domain features of the enzyme and the nature of the flavin environment is available, and will be discussed in an article by $R$. Hille and T. Nishino.

Bacterial enzymes involved in the degradation of unactivated aromatic compounds, such as benzene and catechol, involve both iron-containing dioxygenases and flavoproteins which mediate reduction of the dioxygenase by $\mathrm{NAD}(\mathrm{P}) \mathrm{H}$. One example of this group is phthalate dioxygenase reductase, which contains both FAD and an iron sulfur center. The structure of this enzyme has been solved recently, and will be described along with extensive mechanistic studies in an article in this series by G. T. Gassner, M. L. Ludwig, and D. P. Ballou.

\section{FLAVOENZYMES OF UNKNOWN FUNCTION}

Paradoxically, the flavoprotein discovered first, the old yellow enzyme of brewer's bottom yeast, is difficult to classify both in terms of its physicochemical characteristics, which do not coincide consistently with those of any of the other flavoenzyme classes, and in terms of its physiological function, which is still uncertain. Nevertheless, much is known about the enzyme, including its crystal structure (18). Recent information about this interesting and recalcitrant enzyme, including its interaction with steroids, will be discussed in an article by P. A. Karplus, K. M. Fox, and V. Massey.

\section{UTILITY OF ARTIFICIAL FLAVINS AND STEREOCHEMISTRY OF FLAVOENZYME REACTIONS}

Reference has been made previously to information about flavoprotein structure and mechanism that has been derived from studies where the native FMN or FAD has been removed and replaced by artificial flavins having chemically reactive or environmentally sensitive substituents. Such studies were historically quite important in the absence of structural information from $\mathrm{X}$-ray crystallography. In general, the conclusions reached previously have been substantiated by subsequent crystal structures. An excellent illustration of this is in the determination of whether the interaction of substrate with a particular flavoprotein involved interaction with the si- or the re-face of the flavin $(19,20)$. The principle was based on the known crystal structure of glutathione reductase, where it was shown that the pyridine nucleotide substrate interacted with the re-face of the flavin. By substitution of the native FAD of medium chain acyl-CoA dehydrogenase with 8-hydroxy-5-deaza FAD and reduction by $\mathrm{NaB}^{3} \mathrm{H}_{4}$, it was found that the tritium was incorporated stereospecifically into the reduced flavin, being removed quantitatively on reoxidation with enoyl-CoA. The same reduced flavin, upon incorporation into apoglutathione reductase, also showed quantitative removal of the tritium label on interaction with NADP, thus establishing the stereochemistry of acyl CoA/enoyl-CoA interaction with acyl-CoA dehydrogenase as specific for the reface of the 
flavin. This conclusion was confirmed by the recent X-ray structure of the enzyme (21). The stereochemistry of substrate flavin interaction with a number of enzymes has been determined by this method, and in all cases the conclusions have been confirmed by subsequent crystal structures of the enzyme or those of closely related enzymes. Even when the crystal structure has been solved, it is not always possible to locate a substrate binding site. A good example is $p$ hydroxybenzoate hydroxylase, where the binding site of NADPH could not be identified crystallographically (11) but where the flavin replacement studies clearly demonstrated its interaction with the re-face of the flavin.

Although the use of artificial flavins in providing information about flavin-protein interactions can be considered somewhat redundant by the determination of protein structures by X-ray crystallography, I believe that it will continue to play an important role in flavoenzyme research because it provides information concerning structure in solution as opposed to that in a crystal, and so will serve as a valuable adjunct in providing information on conformational flexibility and its importance in catalysis.

\section{REFERENCES}

1. Massey, V., and Hemmerich, P. (1980) Active site probes of flavoproteins. Biochem. Soc. Transactions 8, 246-257

2. Massey, V. (1994) Activation of molecular oxygen by flavins and flavoproteins. J. Biol. Chem. 269, 22459-22462

3. Ghisla, S., and Massey, V. (1986) New flavins for old: artificial flavins as active site probes of flavoproteins. Biochem. J. 239, 1-12

4. Lindqvist, Y. (1989) Refined structure of spinach glycolate oxidase at $2 \AA$ resolution. J. Mol. Biol. 209, 151-166

5. Hecht, H. J., Kalisz, H. M., Hendle, J., Schmid, R. D., and Schomburg, D. (1993) Crystal structure of glucose oxidase from Aspergillus niger refined at 2.3 A resolution. J. Mol. Biol. 229, 153-172

6. Vrielink, A., Lloyd, L. F., and Blow, D. M. (1991) Crystal structure of cholesterol oxidase from Brevibacterium sterolicum refined at $1.8 \AA$ resolution. J. Mol. Biol. 219, 533-554

7. Ludwig, M. L., and Luschinsky, C. L. (1992) Structure and redox properties of clostridial flavodoxin. In Chemistry and Biochemistry of flavoen- zymes (Müller, F., ed) Vol. III pp. 427-466, CRC Press, Boca Raton, Florida

8. Karplus, P. A., Daniels, M. J., and Herriott, J. R. (1990) Structure of ferredoxin-NADP ${ }^{+}$reductase: prototype for a structurally novel flavoenzyme family. Science 251, 60-66

9. Ghisla, S., Thorpe, C., and Massey, V. (1984) Mechanistic studies with general acyl-CoA dehydrogenase and butyryl-CoA dehydrogenase: evidence for the transfer of the $\beta$-hydrogen to the flavin $N(5)$-position as a hydride. Biochemistry 23, 3154-3161

10. Mizzer, J. P., and Thorpe, C. (1982) Stabilization of the red semiquinone form of pig kidney general acyl-CoA dehydrogenase by acyl-CoA derivatives. In Flavins and Flavoproteins (Massey, V., Williams, C. H., Jr. eds) pp. 637-640 Elsevier-North Holland

11. Entsch, B., and van Berkel, W. J. H. (1995) Structure and mechanism of $p$-hydroxybenzoate hydroxylase. FASEB J. 9, 000-000

12. Williams, G H., Jr. (1992) Lipoamide dehydrogenase, glutathione reductase, thioredoxin reductase and mercuric ion reductase - a family of flavoenzyme transhydrogenases. In Chemistry and Biochemistry of Flavoenzymes (Müller, F., ed) Vol. III, pp. 121-211, CRC Press, Boca Raton, Florida

13. Claiborne, A., Miller, H., Parsonage, D., and Ross, R. P. (1993) Protein-sulfenic acid stabilization and function in enzyme catalysis and gene regulation. FASEB J. 7, 1483-1490

14. Mathews, F. S. (1991) New flavoenzymes. Curr. Opin. Struct. Biol. 1, 954-967

15. Xia, Z-X., and Mathews, F. S. (1990) Molecular structure of flavocytochrome $b_{2}$ at $2.4 \AA$ resolution. J. Mol. Biol 212, 837-863

16. Lederer, F. (1992) Flavocytochrome $b_{2}$. In Chemistry and Biochemistry of Flavoenzymes (Müller, F., ed) Vol. III, pp. 153-242, CRC Press, Boca Raton, Florida

17. Narhi, L. Q, and Fulco, A. J. (1986) Characterization of a self-sufficient 119000 dalton cytochrome P-450 monooxygenase induced by barbiturates in Bacillus megatherium. J. Biol. Chem. 261, 7160-7169

18. Fox, K. M., and Karplus, P. A. (1994) Old yellow enzyme at $2 \AA$ resolution: overall structure, ligand binding and comparison with related flavoproteins. Siructure 2, 1089-1105

19. Manstein, D. J., Pai, E. F., Schopfer, L. M., and Massey, V. (1986) Absolute stereochemistry of flavins in enzyme-catalyzed reactions. Biochemistry 25, 6807-6816

20. Manstein, D. J., Massey, V., Ghisla, S., and Pai, E. F. (1988) Stereochemistry and accessibility of prosthetic groups in flavoproteins. Biochemistry 27, 2300-2305

21. Kim, J-J. P., Wang, M., and Paschke, R. (1993) Crystal structures of medium chain acyl-CoA dehydrogenase from pig liver mitochondria, with and without substrate. Proc. Nath Acad. Sci. USA 90, 7523-7527

\title{
THE 1996 MIAMI BIO/TECHNOLOGY WINTER SYMPOSIUM
}

\author{
Advances in Gene Technology \\ Therapeutic Strategies in Molecular Medicine \\ February 10-14, 1996
}

New Location:

Marina Marriott

Fort Lauderdale, Florida, USA

For further information contact:

MBWS Office (M823)

P. O. Box 016129

Miami, FL 33101, USA

Phone: 1-800-MIA-GENE (1-800-642-4363) or 1-305-243-3597

Fax: 1-305-324-5665; e-mail: mbws@mednet.med.miami, edu 